\title{
Modelling Gelation Time of Organically Cross-linked Water-shutoff Systems for Oil Wells
}

\section{Marfo SA ${ }^{1}$, Appah $\mathrm{D}^{2}$, Joel $\mathrm{OF}^{3}$ and Ofori-Sarpong $\mathrm{G}^{4}$}

${ }^{1}$ World Bank African Centre of Excellence in Oilfield Chemicals Research, IPS, Uniport, Nigeria : Petroleum Engineering Department, University of Mines and Technology, Tarkwa, Ghana

${ }^{2}$ Department of Gas Engineering, Uniport, Nigeria

${ }^{3}$ Centre for Petroleum Research and Training, IPS, Uniport, Nigeria

${ }^{4}$ Minerals Engineering Department, University of Mines and Technology, Tarkwa, Ghana

\begin{abstract}
Water production is one of the major challenges in the petroleum industry, especially brown fields and water drive reservoirs. Water production can weaken the cementation of sand grains, thereby rendering formations partially or completely unconsolidated. This in turn initiates fines migration and aggravates safety concerns. The gelation time is an important characteristic of water-shutoff systems and it is influenced by different parameters. The gelation time gives an indication of the time required for a gel to transit from free flowing fluid to solid or semi-solid gel making it difficult to pump. Organically cross-linked gels have numerous applications in the industry such as shutoff systems. They are used to control water and gas production in oil wells. The effect of cross-linker concentration, temperature and brine concentration on the gelation time of an organically crosslinked system was studied. Based on the experimental results a mathematical model was developed for predicting the gelation time of the water-shutoff system. The results showed that temperature had the highest impact on the gelation time of the water-shutoff system with an effect estimate of (-2.292). The brine concentration of the mix water recorded the lowest impact with an effect estimate of 0.2083 and the interaction between cross-linker concentration and brine concentration of mix water had neutral impact. A predictable and effective water-shutoff system has been developed with an excellent initial viscosity which can easily be pumped and applied to solve water production and its associated problems. The gelation time of organically crosslinked water-shutoff system can be optimised in water control operations using this model and the effect estimates of these parameters.
\end{abstract}

Keywords: Water-shutoff system; Gelation time; Polymer; Cross linker; Brine; Modelling

\section{Introduction}

During petroleum production, an acceptable amount of water production is expected and can even be beneficial in the initial stages of the life of a well. This water however can be problematic when it is in excess [1]. Excessive water production is one of the major challenges facing the oil and gas industry [2-4]. Water production affects the economics of producing wells as it has to be treated and disposed of in an environmentally friendly manner at an additional cost $[3,5]$. Produced water cost the petroleum industry about $\$ 45$ billion in 2002 $[5,6]$, and this could be on the increase with development of additional wells. High water cut comes with its associated problems such as corrosion, sand production, scale formation and loss of productivity [1,7-9]. This occurrence is common with mature fields [10].

Excessive water production can be the result of the natural depletion of a reservoir where either naturally or artificially active water drive has swept away most of the oil that the reservoir can produce. Causes of water production can either be completion-or reservoir-related. Many researchers $[1,11,12]$ identified the completion-related issues as casing leaks, channel behind casing, completion into water; and the reservoirrelated mechanisms as coning or cusping, fractures or faults, and stimulation out of zone, among others.

Excessive water production can be controlled either by mechanical or chemical means $[6,13]$. The mechanical methods include using hardware or cement as mechanical seals or isolations to control water production, coning control through draw-down reduction and coproduction and downhole separation. These mechanical methods such as casing and tubing patches, bridge plugs and cement squeeze can work but not for all types of water production mechanisms. This results in searching for other means which are more efficient and can penetrate the formations to either partially or completely block water producing zones.

The search for efficient chemical means of water control over the decades has evolved with different chemicals; both organic and inorganic being developed. Researchers [1,6,13-16] have identified polymer gels for near wellbore area (organically or inorganically crosslinked) and microgels for deep profile modification (Bright water and Methylene bisacrylamide Aggregates) as the broad categories of chemicals for water sealants in wells producing excessive and unwanted water. According to Watters, Kabir, Sydansk, these broad categories include; inorganic gels, resins or elastomers, monomer based systems, polymer gels, viscous systems, bio-polymers and foam gel among others $[1,13,17,18]$.

The gelation time of water-shutoff systems is a function of the concentrations of polymer (base gel), cross-linker concentration, temperature, salinity of mixing water and $\mathrm{pH}$ of the fluid system among others [6]. Temperature has an important effect on the rheological

*Corresponding author: Marfo SA, World Bank African Centre of Excellence in Oilfield Chemicals Research, IPS, Uniport, Nigeria; Petroleum Engineering Department, University of Mines and Technology, Tarkwa, Ghana, E-mail: smarfo@ umat.edu.gh

Receive November 24, 2015; Accepted January 29, 2016; Published February 06, 2016

Citation: Marfo SA, Appah D, Joel OF, Ofori-Sarpong G (2016) Modelling Gelation Time of Organically Cross-linked Water-shutoff Systems for Oil Wells. J Pet Environ Biotechnol 7: 267. doi:10.4172/2157-7463.1000267

Copyright: @ 2016 Marfo SA, et al. This is an open-access article distributed under the terms of the Creative Commons Attribution License, which permits unrestricted use, distribution, and reproduction in any medium, provided the original author and 
properties of polymeric melts, just as it does on polymeric solids. The gelation point of polymers is temperature and time dependent at a determined favourable cross-linker concentration. There is a gradual rise in viscosity followed by an asymptotic increase to infinity as the gel point is approached Marfo [19]. Al-Muntasheri [14] developed correlation for gelation time as a function of temperature for watershutoff systems. These equations showed that increasing the temperature shortened the gelation time of organically crosslinked gels. Reddy [9] developed correlations for gelation time as a function of degree of salinity of mixing fluid for $\mathrm{KCl}$ and $\mathrm{NaCl}$. Their findings indicated a linear relation between gelation time and the salt concentration. It was concluded that increasing degree of salinity increased the gelation time of organically crosslinked water-shutoff systems. El-Karsani, Reddy $\mathrm{BR}, \mathrm{Al}$-Muntasheri and Das concluded that increasing the polymer and cross-linker concentrations resulted in a decrease in the gelation time of water-shutoff systems $[4,6,9,14,20]$.

The research findings reported so far examined one parameter at a time in developing correlations as a function of gelation time. This paper, however, explains how the interaction between three parameters; cross-linker concentration, temperature and brine concentration impact on the gelation time of organically crosslinked water-shutoff systems. The parameter effect estimates and the interaction between these parameters with their corresponding impacts on gelation time were studied using factorial design and are presented in this paper. Efficient model with excellent gelation time prediction and gelation time equation as a function of temperature, mix water salinity and cross-linker concentration are developed and presented.

\section{Materials and Methods}

\section{Materials}

Organically crosslinked system comprising of acrylamide/acrylate copolymer crosslinked with polyamine was used in this experiment and these were in aqueous form. Different formulations were designed using $2 \%$ and $5 \%$ potassium chloride $(\mathrm{KCl})$ brine as the mix water. The concentrations of the polyamine (cross-linker) used were $6 \mathrm{wt} \%$, $9 \mathrm{wt} \%$ and $12 \mathrm{wt} \%$ corresponding to $60 \mathrm{gal} / 1000 \mathrm{gal}, 90 \mathrm{gal} / 1000 \mathrm{gal}$ and 120 $\mathrm{gal} / 1000 \mathrm{gal}$ of the base polymer respectively. These were formulated at a constant base polymer of $350 \mathrm{gal} / 1000 \mathrm{gal}$ representing $35 \mathrm{wt} \%$. All the chemicals used are American Chemical Standard (ACS) grade. The $\mathrm{pH}$ of the solutions was measured using HI991003 $\mathrm{pH}$ meter. The gelation times of the various formulations were determined at preset temperatures using Fann 35 Viscometer.

\section{Procedure}

The mix fluid and the required amount of polymer were stirred thoroughly in a blender. The $\mathrm{pH}$ of the solution was then measured. The required amount of cross-linker was added to the solution and stirred thoroughly, and the $\mathrm{pH}$ of the solution was measured. The apparent viscosity of the sample was monitored and poured into 8 $\mathrm{oz}$ glass jar and placed in water preset to the test temperature. The apparent viscosity was monitored at regular interval (30 minutes) with a Fann 35 viscometer, equipped with F1 spring, B1 bob and R1 rotor @ shear rate of $511 \mathrm{~s}^{-1}$

\section{Results and Discussion}

The water-shutoff system was observed to be alkaline as the $\mathrm{pH}$ of the system was in the range of 9.91 to 10.52 . Addition of the polymer to the different mix fluid concentrations resulted in $\mathrm{pH}$ in the range of 5 to 8 indicating a slightly acidic medium. Upon adding the cross-linker, the system's $\mathrm{pH}$ changed from 9.91 to 10.52 , indicating that crosslinker is a strong alkaline. The water-shutoff system developed has similar $\mathrm{pH}$ (approximately 10) with other conformance sealants $[5,10]$. Maintaining the $\mathrm{pH}$ of the system in slightly alkaline medium enhances the hydration process of polymers. This indicates that adjusting the $\mathrm{pH}$ to acidic medium will change the gelation time as confirmed by Boye [5]. It is thus advised to minimise the exposure of this system to acidic medium and other contaminants as the $\mathrm{pH}$ of fluids control crosslinker function and polymer hydration [1].

The viscosity build-up of the different formulations is both time and temperature dependent. To study the viscosity build-up of the system with temperature and time, different formulations were prepared and the viscosity measured at regular intervals (30 minutes) until the system gelled. When water-shutoff system was placed in the water bath at the present temperature, the apparent viscosity of the system decreased slightly from what was obtained at room temperature before it started building-up, and this is due to the thinning effect of the polymer and cross-linker. The viscosity build-up with time for water-shutoff design systems at different brine concentration and formulations are shown in Figures 1 and 2.

\section{Temperature and cross-linker effect}

The temperature and cross-linker effect on water-shutoff design for brine $(2 \%$ and $5 \% \mathrm{KCl})$ are shown in Figures 3 and 4 respectively. The effect of cross-linker concentration on gelation time of the system was determined by varying the cross-linker concentrations $(6,9$ and $12 \mathrm{wt} \%)$ with all other parameters kept constant. The effect of crosslinker concentrations was determined at temperatures $140^{\circ} \mathrm{F}$ and 190 ${ }^{\circ} \mathrm{F}$. Cross-linker concentrations, salinity of mix water and temperature have effect on the gelation time of water-shutoff systems. Increasing

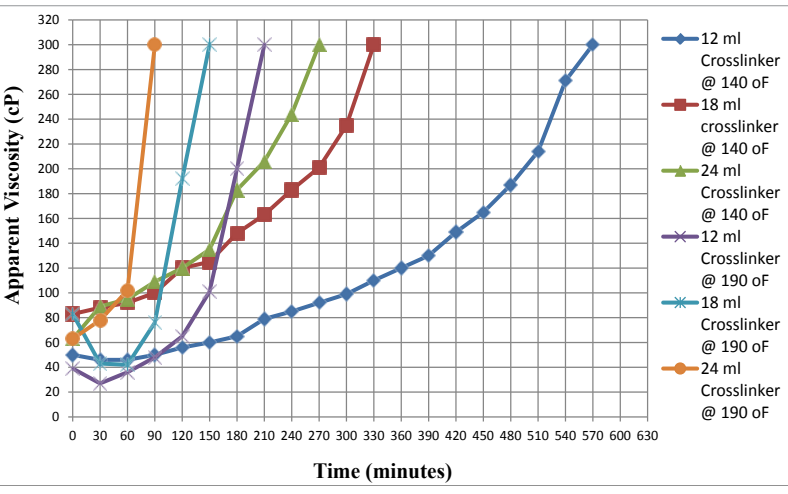

Figure 1: Viscosity build-up for $2 \% \mathrm{KCl}$ water-shutoff design.

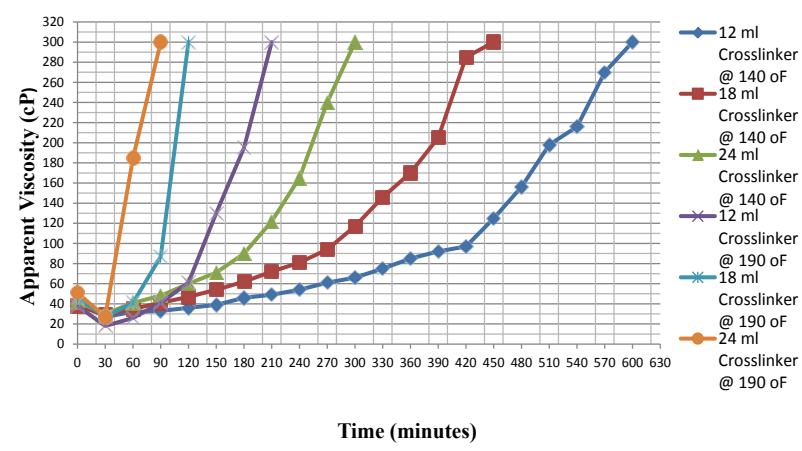

Figure 2: Viscosity build-up for $5 \% \mathrm{KCL}$ water-shutoff design. 


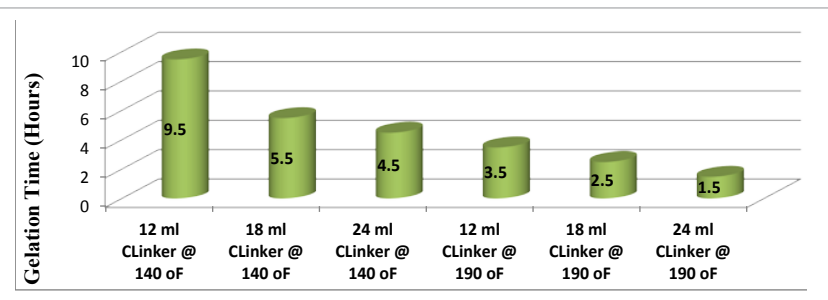

Figure 3: Temperature and crosslinker effect on gel time of water-shutoff for $2 \% \mathrm{KCl}$.

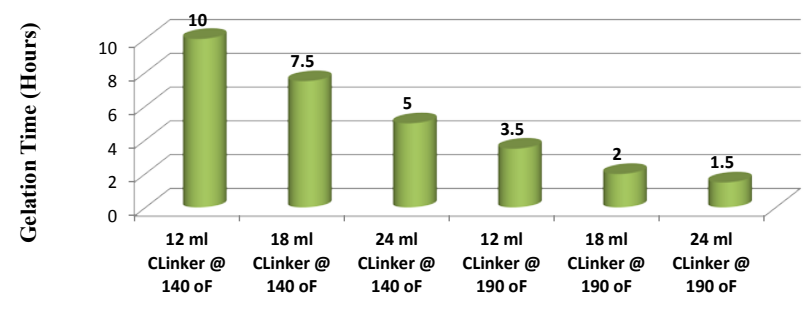

Figure 4: Temperature and crosslinker effect on gel time of water-shutoff for $5 \% \mathrm{KCl}$.

the cross-linker concentration shortens the gelation time and this occurs in all the formulations, at different brine concentrations and test temperatures. This is as a result of creation of more sites for crosslinking $[1,10]$, leading to hydration and enhancing rate of reaction. So the more concentrated the cross-linker, the faster the rate of reaction and the shorter the gelation time as shown in the Figures 3 and 4 .

Temperature had the greatest impact on the gelation time, and this was determined by measuring the gelation time at different temperatures $\left(140^{\circ} \mathrm{F}\right.$ and $\left.190^{\circ} \mathrm{F}\right)$. Irrespective of the cross-linker and brine concentration, an increase in temperature drastically decreased the gelation time with the greatest impact occurring at lower crosslinker concentrations. This is predictive of gel systems indicating endothermic reaction as increase in temperature decreases gelation time [14]. An increase in temperature is a good platform for viscosity of gel systems to build-up as crosslinking density is increased resulting in increased rate of reaction and thereby shortening the gelation time. Other explanations for this could be increased hydrolysis of the base polymer at higher temperatures that increased the rate of crosslinking, resulting in increase in molecular mobility and creating more crosslink sites for reaction [10] among others. Therefore in the gelation process, the rate of crosslinking is accelerated with increased temperature, and the gelation time is decreased.

\section{Brine concentration effect}

Increasing brine concentration increased the gelation time of the system, and this impact was greater at lower temperatures. At $140{ }^{\circ} \mathrm{F}$, $5 \%$ brine gave a gelation time of 7.5 hours whereas decreasing brine concentration to $2 \%$ shortened the time to 5.5 hours for the same formulation. At a higher temperature of $190^{\circ} \mathrm{F}$, the effect of brine concentration on gelation time diminished as different concentrations gave the same gelation time value for the same formulation. The effect of brine concentration $(2 \% \mathrm{KCl}$ and $5 \% \mathrm{KCl})$ on gelation time of watershutoff design at temperatures $140{ }^{\circ} \mathrm{F}$ and $190^{\circ} \mathrm{F}$ are shown in Figures 5 and 6 respectively. From Figure 5, it is observed that increasing brine concentration from $2 \%$ to $5 \% \mathrm{KCl}$ at $140{ }^{\circ} \mathrm{F}$ increased the gelation time for the system and this was noticed in all the formulations. This phenomenon can be attributed to the effect of brine on the hydrolysis of polymer. Brine is known to cause shrinkage of polymer hydration thereby reducing the crosslinking sites available for reaction leading to slow reaction rate, and thus the elongation in the gelation time.
This explains the trend of increase in gelation time when the brine concentration was increased from $2 \%$ to $5 \% \mathrm{KCl}$ mixed fluid. However, at $190^{\circ} \mathrm{F}$ the effect of brine concentration was not significant as it did not affect the gelation time of the designs. The possible explanation though not conclusive could be the interactive effect of high temperature and brine concentration. The only exception was when $18 \mathrm{ml}$ of cross-linker was used. This did not follow the trend seen at $140^{\circ} \mathrm{F}$ where increasing brine concentration increased the gelation time (Figures 5 and 6).

This is in line with the findings of researchers $[1,6,10,20]$ as the gelation time of the developed system is influenced by factors such as temperature, salinity of mix water, $\mathrm{pH}$, cross-linker and base polymer concentration, among others. The effect of cross-linker concentration on gelation time for $2 \%$ and $5 \% \mathrm{KCl}$ with predictive equations for temperatures at $140{ }^{\circ} \mathrm{F}$ and $190{ }^{\circ} \mathrm{F}$ are shown in Figures 7 and 8 respectively. These equations can be used to predict the gelation time at the given temperatures and at constant base polymer concentration. The equations make it easier to determine the required crosslinker concentration to achieve the desired gelation time at a given temperature and constant polymer base concentration.

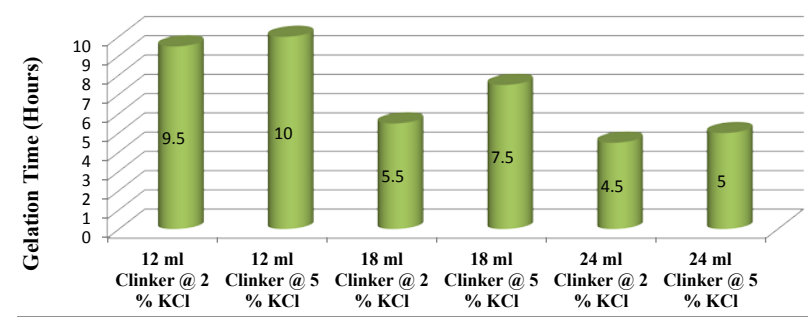

Figure 5: Effect of brine on gelation time for water-shutoff design @ $140^{\circ} \mathrm{F}$.

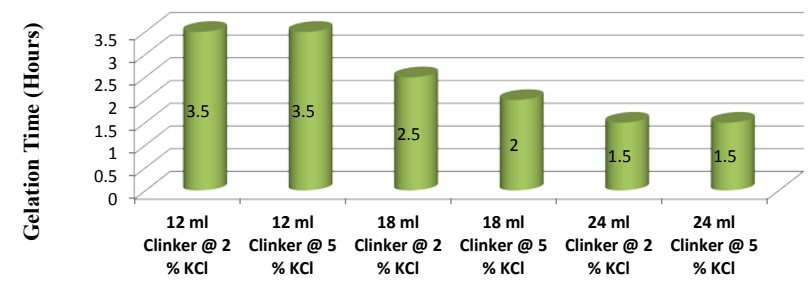

Figure 6: Effect of brine on gelation time for water-shutoff design @ $190^{\circ} \mathrm{F}$.

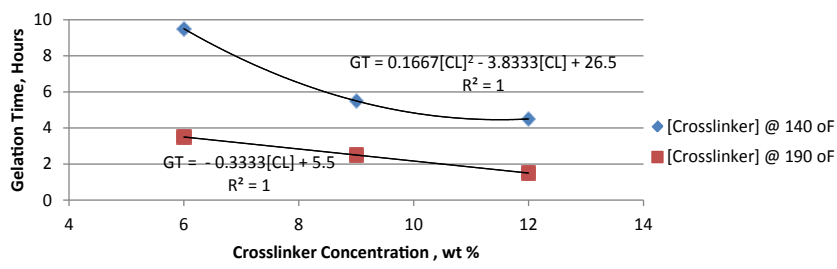

Figure 7: Effect of cross-linker concentration on gelation Time for $2 \% \mathrm{KCl}$.

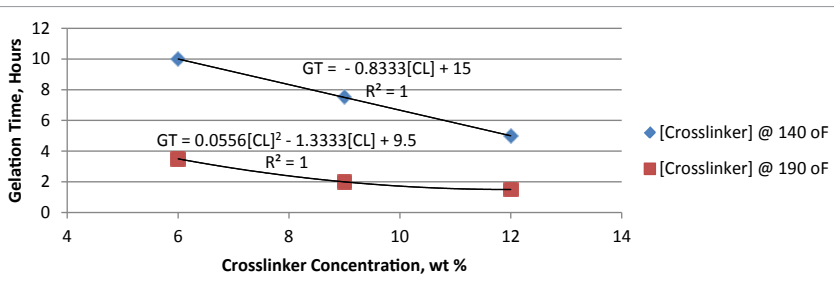

Figure 8: Effect of cross-linker concentration on gelation time for $5 \% \mathrm{KCl}$. 
To study the interactive effect of the design parameters; temperature, salinity of mix water, and cross-linker concentration on the gelation time of the water-shutoff system, a factorial design was performed. The results obtained from the experiment were modelled using JMP; a statistical analysis software. The summary of fit and the analysis of variance (ANOVA) generated for the water-shutoff model developed are shown in Tables 1 and 2 respectively. The R-Square which is the ratio of the sum of squares of the model to the sum of squares of the total obtained from the model, measures how well a model will predict new data. The R-Square obtained for the water-shutoff system is 0.982 meaning the model is a good predictor and expected to explain about $98 \%$ of the variability of the gelation time of the water-shutoff system in a new data designed using this system. The corresponding R-square adjusted for this model is about $96 \%$. The R-Square adjusted $\left(R^{2}\right.$ adj) is a statistic adjusted for the size of the model which takes care of the number of factors considered in a model.

The parameter estimates for the model are shown in Table 3. The highest impact for the model is from the temperature parameter $(-2.292)$ with a negative value indicating an inverse relationship between it and the response variable. The mix water salinity $(0.20833)$ gave the least impact on the model, with the interaction between cross-linker concentration and mix water salinity having neutral impact on the model.

The residual by predicted gelation time is shown in Figure 9. There are residuals for this model and this confirms the $98 \%$ predictability for the model.

The interaction between the design parameters and the effect on the gelation time for the model is shown in the cube plot in Figure 10. The cube plot can be used in determining which parameters to control to achieve a desired gelation time. Additionally, it can be used as an optimisation tool. The gelation times are superimposed on the eight corners of the box and these values correspond to the assigned values for the designed parameters. It can be seen that the longest gelation time (10 hours) for the system occurred when the temperature and cross-linker concentration parameters are at the lowest level and the

\begin{tabular}{|c|c|}
\hline R-Square & 0.982498359 \\
\hline R-Square Adj & 0.96149639 \\
\hline Root Mean Square Error & 0.577350269 \\
\hline Mean of Response & 4.708333333 \\
\hline Observations (or Sum Weights) & 12 \\
\hline
\end{tabular}

Table 1: Summary of fit for water-shutoff model.

\begin{tabular}{|l|l|l|l|l|l|}
\hline Source & DF & $\begin{array}{l}\text { Sum of } \\
\text { Squares }\end{array}$ & $\begin{array}{l}\text { Mean } \\
\text { Square }\end{array}$ & F Ratio & Prob > F \\
\hline Model & 6 & 93.5625 & 15.5938 & 46.7813 & 0.0003 \\
\hline Error & 5 & 1.666667 & 0.3333 & & \\
\hline C. Total & 11 & 95.229167 & & & \\
\hline
\end{tabular}

Table 2: Analysis of variance for water-shutoff model.

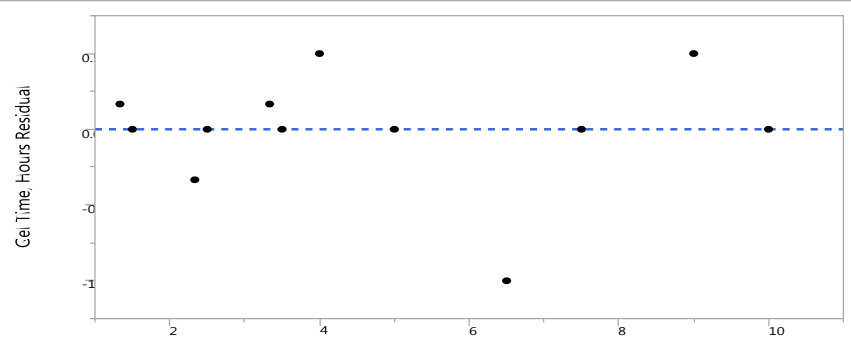

Figure 9: Residual by predicted plot for the model.

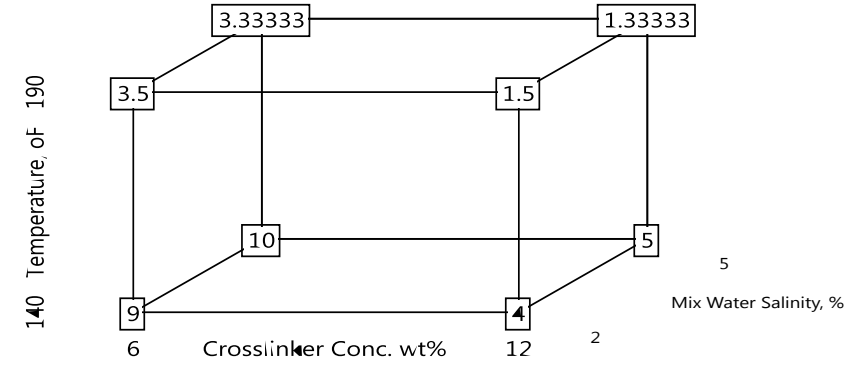

Figure 10: Cube plot for the model.

mix water salinity is at the highest level. For the shortest gelation time (1.333 hours) it occurred at the highest point for all the design factors; temperature, cross-linker concentration and mix water salinity.

The two dimensional contour plots for temperature vs crosslinker concentration, temperature vs mix water salinity and crosslinker concentration vs mix water salinity are shown in Figures 11-13 respectively. These plots predict the behaviour of the gelation time in the various regions created by the design parameters in the model developed. For Figure 11, the maximum gelation time is reached around $140{ }^{\circ} \mathrm{F}$ and $6 \mathrm{wt} \%$ of cross-linker concentration whereas the minimum is attained around $190{ }^{\circ} \mathrm{F}$ and $12 \mathrm{wt} \%$ of cross-linker. The longest gelation time for Figure 12 occured around $140{ }^{\circ} \mathrm{F}$ and $5 \%$ mix water salinity and the shortest gelation time took place around $185^{\circ} \mathrm{F}$ irrespective of the degree of salinity of mix water. For Figure 13, the maximum gelation time is recorded around $5 \%$ mix water salinity and $6 \mathrm{wt} \%$ of cross-linker whereas the minimum took place around $11 \mathrm{wt} \%$ of cross-linker and $2 \%$ of mix water salinity.

A predictive equation for gelation time GT (hours) was developed from the model (Equation 1). The water-shutoff model's equation has an R-Square of $98 \%$ and the design parameters are temperature $\mathrm{T}\left({ }^{\circ} \mathrm{F}\right)$, salinity of mix water S (\%) and cross-linker concentration C (wt\%).

\section{Conclusions}

$$
G T=38.4333-\frac{13}{75} T+\frac{19}{30} S-\frac{67}{30} C+\frac{1}{100} T^{*} C-\frac{1}{300} T^{*} S[1]
$$

From the research, the following conclusions could be made:

a. Effective and efficient predictable water-shutoff system using an organically crosslinked polymer with an excellent initial viscosity has been designed. This is indicative of a system that can easily be pumped into the formation matrix.

b. The performance in $2 \%$ and $5 \% \mathrm{KCl}$ brine mix fluid and in different formation temperatures has been tested, and the results indicate that the system can be designed to achieve its desired function in different formations.

c. Temperature, cross-linker concentration and mix fluid salinity and the interaction between these parameters have effect on the gelation time of water-shutoff systems. The research revealed that the longest gelation time ( 10 hours) for the model occurred when temperature and cross-linker concentration were at the lowest levels and the mix water salinity was at the highest level. The shortest gelation time (1.333 hours) for the system occurred at the highest levels for all the design parameters.

d. Temperature recorded the highest impact on the gelation time with an effect estimate of (-2.292). This was followed by 
Citation: Marfo SA, Appah D, Joel OF, Ofori-Sarpong G (2016) Modelling Gelation Time of Organically Cross-linked Water-shutoff Systems for Oil Wells. J Pet Environ Biotechnol 7: 267. doi:10.4172/2157-7463.1000267

Page 5 of 6

\begin{tabular}{|c|c|c|c|c|}
\hline Term & Estimate & Std Error & t Ratio & Prob $>|t|$ \\
\hline Temperature, ${ }^{\circ} \mathrm{F}(140,190)$ & -2.291666667 & 0.166666667 & -13.75 & $<0.0001$ \\
\hline Crosslinker Conc. wt\% & -0.583333333 & 0.068041382 & -8.57 & 0.0004 \\
\hline (Crosslinker Conc. wt\%-9)*Temperature, ${ }^{\circ} \mathrm{F}$ & 0.25 & 0.068041382 & 3.67 & 0.0144 \\
\hline Temperature, ${ }^{\circ} \mathrm{F}^{\star}$ Mix Water Salinity, \% & -0.291666667 & 0.166666667 & -1.75 & 0.1405 \\
\hline Mix Water Salinity, \%(2.5) & 0.208333333 & 0.166666667 & 1.25 & 0.2666 \\
\hline$($ Crosslinker Conc. wt\%-9)*Mix Water Salinity, \% & 0 & 0.068041382 & 0 & 1 \\
\hline
\end{tabular}

Table 3: Parameter estimate for water-shutoff model.

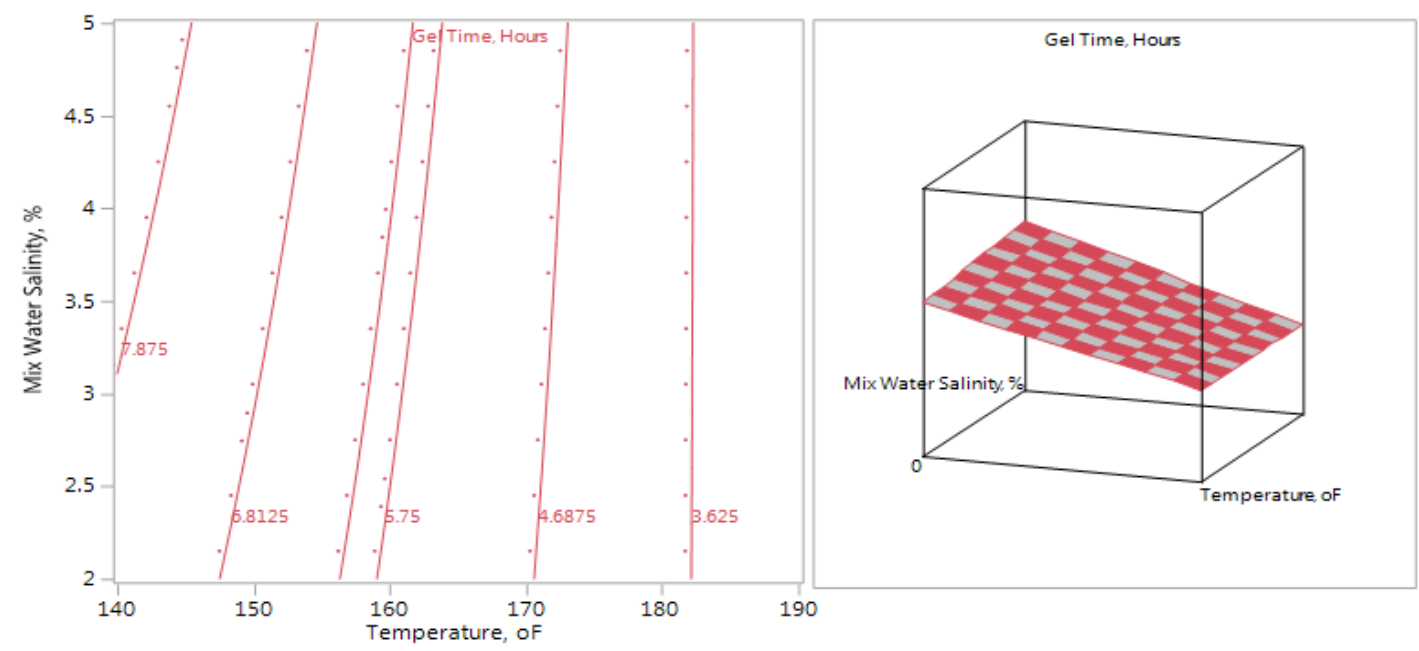

Figure 11: Contour plot of temperature vs. crosslinker concentration for model.

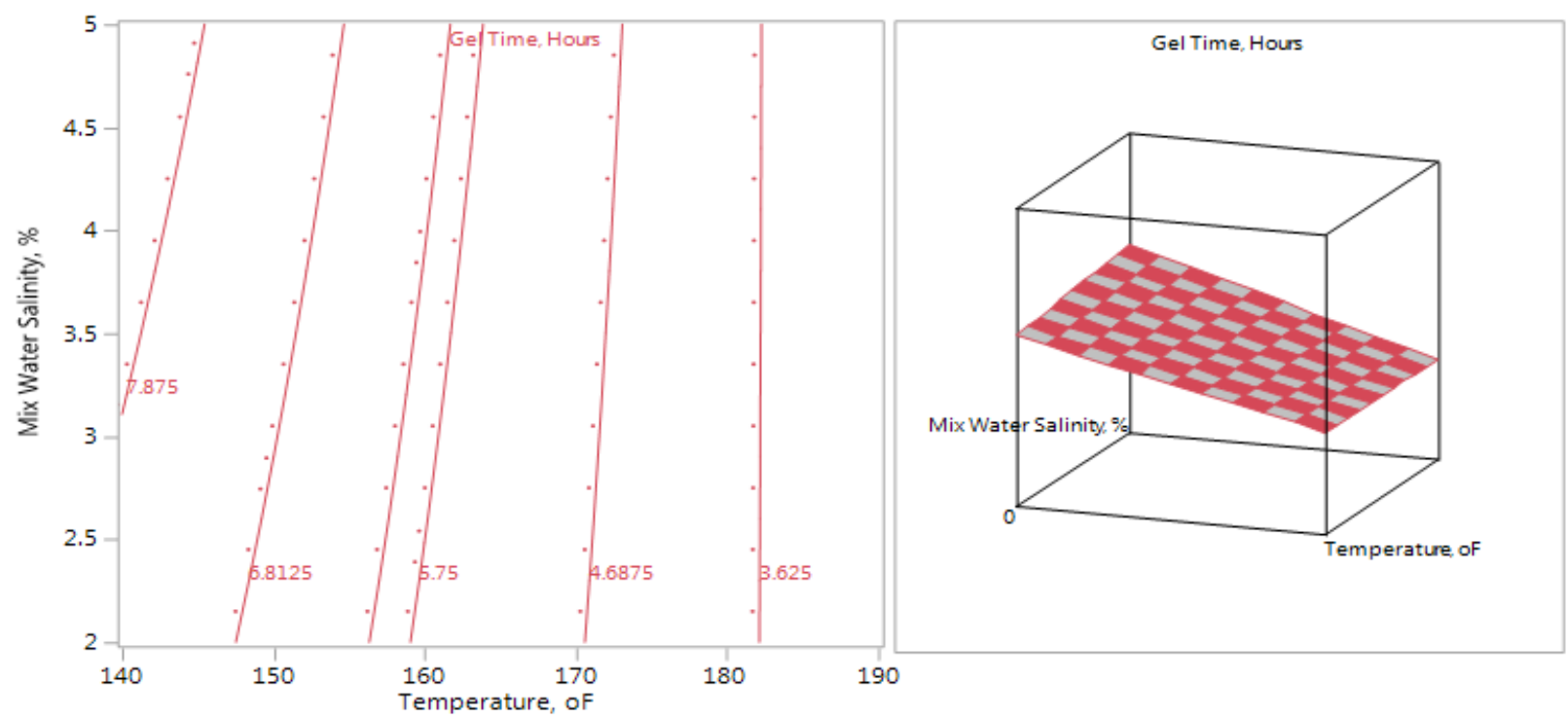

Figure 12: Contour plot of temperature vs.mix water salinity for model.

cross-linker concentration (-0.5833), temperature - mix water salinity interaction $(-0.2917)$, cross-linker concentration temperature interaction (0.25) and mix water salinity (0.2083). The interaction between the cross-linker concentration and mix water salinity had neutral impact on the gelation time.

e. An efficient and good predictor model with an R-square of about $98 \%$ and equations expressing the gelation time as a function of temperature, cross-linker concentration and mix water salinity has been developed.

\section{Acknowledgements}

The authors wish to express their thanks to the World Bank for the offer of PhD scholarship at the World Bank African Centre of Excellence, Institute of Petroleum Studies, University of Port Harcourt, Nigeria and University of Mines and Technology, Tarkwa, Ghana for financially supporting this research work. 
Citation: Marfo SA, Appah D, Joel OF, Ofori-Sarpong G (2016) Modelling Gelation Time of Organically Cross-linked Water-shutoff Systems for Oil Wells. J Pet Environ Biotechnol 7: 267. doi:10.4172/2157-7463.1000267

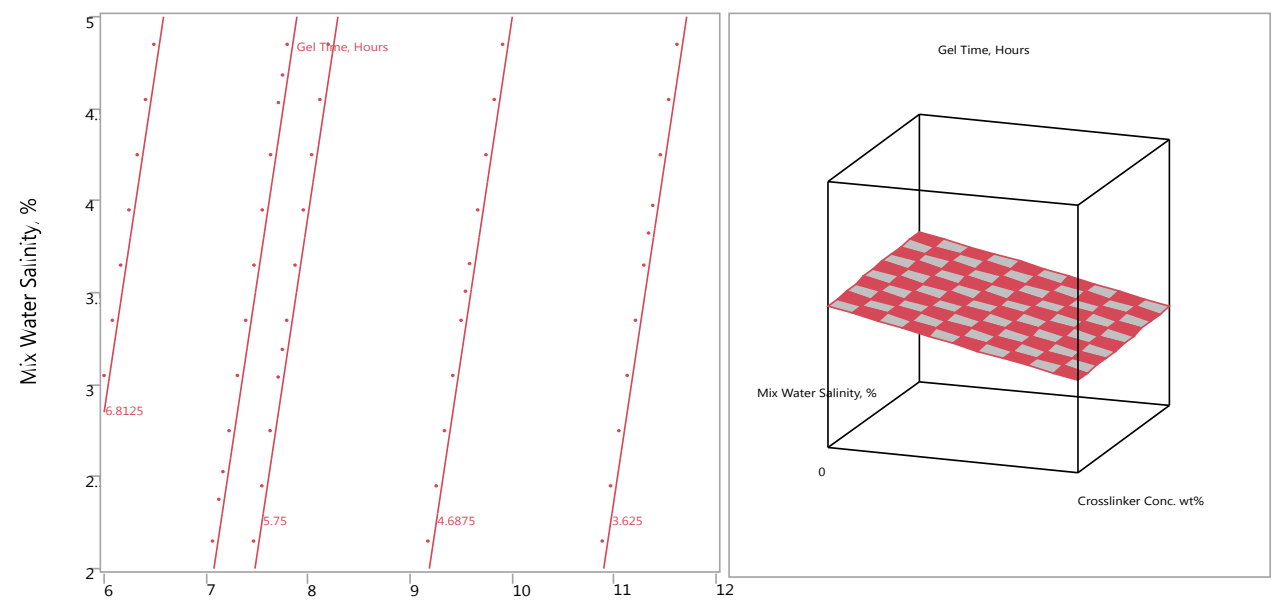

Figure 13: Contour plot of crosslinker concentration vs. mix water salinity for model.

\section{References}

1. Economides MJ, Watters L (1997) Petroleum well construction. John Wiley and Sons.

2. Lei G, Li L, Nasr-El-Din HA (2010) New gel aggregates for water shut-off treatments. Proceedings of the SPE Improved Oil Recovery Symposium, Tulsa, Oklahoma, USA.

3. Salgaonkar L, Das P (2012) Laboratory evaluation of organically crosslinked polymer for water shutoff in high-temperature well applications. Proceedings of the SPE International Petroleum Conference and Exhibition, Kuwait.

4. Vasquez JE, Tuck D (2015) Environmentally acceptable porosity-fill sealant systems for water and gas control applications. Proceedings of the SPE HSE and Sustainability Conference, Colombia.

5. Boye B, Rygg A, Jodal C, Klungland I (2011) Development and evaluation of a new environmentally acceptable conformance sealant. Proceedings of the SPE European Formation Damage Conference, Noordwijk, The Netherlands.

6. El-Karsani KS, Al-Muntasheri GA, Hussein IA (2014) Polymer systems for water shutoff and profile modification: A review over the last decade.

7. Van der Hoek JE, Botermans W, Zitha PLJ (2001) Full blocking mechanism of polymer gels for water control. Proceedings of the SPE European Formation Damage Conference, The Hague, Netherlands.

8. You Q, Tang $Y$, Wang B, Zhao $F$ (2011) Enhanced oil recovery and corrosion inhibition through a combined technology of gel treatment for water shutoff and corrosion inhibitor huff \& puff in oil well. Procedia Engineering 18: 7-12.

9. Reddy BR, Crespo F, Eoff $L$ (2012) Water shutoff at ultralow temperature using organically crosslinked polymer gels. Proceedings of the SPE Improved Oil Recovery Symposium, Tulsa, Oklahoma, USA

10. El-Karsani KS, Al-Muntasheri GA, Sultan AS, Hussein IA (2014) Gelation of a water-shutoff gel at high pressure and high temperature: Rheological investigation. SPE Journal.
11. Barrufet MA, Burnett D, Macauley J (1998) Screening and evaluation of modified starches as water shutoff agents in fractures and in matrix flow configurations. Proceedings of the SPE Improved Oil Recovery Symposium, Tulsa, Oklahoma.

12. Clegg JD (2007) Production operations engineering: Well production problems - Water control. Petroleum engineering handbook. Lake LW, Richardson, Texas SPE 4: 381-384.

13. Kabir AH (2001) Chemical water \& gas shutoff technology - An overview. Proceedings of the SPE Asia Pacific Improved Oil Recovery Conference, Kuala Lumpur, Malaysia.

14. Al-Muntasheri GA, Nasr-El-Din HA, Zitha PLJ (2008) Gelation kinetics and performance evaluation of an organically crosslinked gel at high temperature and pressure. SPE Journal.

15. Deolarte C, Vasquez J, Sorian E, Santillan A (2009) Successful combination of an organically crosslinked polymer system and a rigid-setting material for conformance control in Mexico. SPE Production \& Operation: 522-529.

16. Reddy BR, Eoff L, Crespo F, Lewis C, (2013) Recent advances in organically crosslinked conformance polymer systems. Proceedings of the SPE International Symposium on Oilfield Chemistry, The Woodlands, Texas, USA.

7. Sydansk RD, Southwell P (2000) More than 12 years of experience with a successful conformance-control polymer gel technology. Proceedings of the SPE AAPG Western Regional Meeting.

18. Sydansk RD, Seright RS (2007) When and where relative permeability modification water-shutoff treatments can be successfully applied. Proceedings of the SPE Symposium Improved Oil Recovery, USA

19. Marfo SA, Appah D, Joel OF, Ofori-Sarpong G (2015) Sand consolidation operations, challenges and remedy. Proceedings of the SPE Nigeria Annual International Conference and Exhibition, Nigeria.

20. Das P, Patil P, Agashe S (2013) Application of relative permeability modifier for sealant diversion. Proceedings of the SPE North Africa Technical Conference and Exhibition, Cairo, Egypt. 